\title{
Stability of functionalized platform molecules on $\mathrm{Au}(111)$
}

Torben Jasper-Tönnies, Igor Poltavsky, Sandra Ulrich, Tobias Moje, Alexandre Tkatchenko, Rainer Herges, and Richard Berndt

Citation: J. Chem. Phys. 149, 244705 (2018); doi: 10.1063/1.5059344

View online: https://doi.org/10.1063/1.5059344

View Table of Contents: http://aip.scitation.org/toc/jcp/149/24

Published by the American Institute of Physics 


\title{
Stability of functionalized platform molecules on $\mathrm{Au}(111)$
}

\author{
Torben Jasper-Tönnies, ${ }^{1, a)}$ Igor Poltavsky, ${ }^{2}$ Sandra Ulrich, ${ }^{3}$ Tobias Moje, ${ }^{3}$ \\ Alexandre Tkatchenko, ${ }^{2}$ Rainer Herges,${ }^{3}$ and Richard Berndt ${ }^{1}$ \\ ${ }^{1}$ Institut für Experimentelle und Angewandte Physik, Christian-Albrechts-Universität zu Kiel, \\ 24098 Kiel, Germany \\ ${ }^{2}$ Physics and Materials Science Research Unit, University of Luxembourg, Luxembourg City L-1511, Luxembourg \\ ${ }^{3}$ Otto-Diels-Institut für Organische Chemie, Christian-Albrechts-Universität zu Kiel, 24098 Kiel, Germany
}

(Received 20 September 2018; accepted 14 November 2018; published online 27 December 2018)

\begin{abstract}
Trioxatriangulenium (TOTA) platform molecules were functionalized with methyl, ethyl, ethynyl, propynyl, and hydrogen and sublimated onto $\mathrm{Au}(111)$ surfaces. Low-temperature scanning tunneling microscopy data reveal that $>99 \%$ of ethyl-TOTA and methyl-TOTA remain intact, whereas $60 \%$ of H-TOTA and $>99 \%$ of propynyl-TOTA and ethynyl-TOTA decompose. The observed tendency toward fragmentation on $\mathrm{Au}(111)$ is opposite to the sequence of gas-phase stabilities of the molecules. Although $\mathrm{Au}(111)$ is the noblest of all metal surfaces, the binding energies of the decomposition products to $\mathrm{Au}(111)$ destabilize the functionalized platforms by 2 to $3.9 \mathrm{eV}(190-370 \mathrm{~kJ} / \mathrm{mol})$ and even render some of them unstable as revealed by density functional theory calculations. Van der Waals forces are important, as they drive the adsorption of the platform molecules. Published by AIP Publishing. https://doi.org/10.1063/1.5059344
\end{abstract}

\section{INTRODUCTION}

The properties and functions of molecules adsorbed to metals crucially depend on the adsorption geometry and the electronic coupling to the substrate. Chemical anchor groups consisting of a molecular subunit or just a single atom are used to determine these parameters for a class of molecules. ${ }^{1-15}$ Recently, platform molecules have been used as anchors to a substrate and to achieve decoupling from neighbor molecules. ${ }^{16,17}$ Extended aromatic platforms are particularly interesting because they adhere to a substrate via physisorption instead of chemisorption. With these platforms lying flat on the substrate, the functional molecule juts out into vacuum. ${ }^{18}$ The electronic coupling at the molecule-surface interface is mediated by an extended $\pi$ system. Accordingly, physisorbed platforms were employed as high-conductance contacts between a metal electrode and molecular wires ${ }^{19,20}$ and $\mathrm{Zn}$-porphyrin molecules. ${ }^{21}$ To reduce the electronic coupling, a spacer may be inserted between the functional molecule and the platform. ${ }^{17,18,22-24}$ Photochemical cleavage of an axial phenyl acetylene from a platform molecule at the ethanol/Au(111) interface was reported. ${ }^{25}$

While $\mathrm{Au}(111)$ is the noblest of all metal surfaces, ${ }^{26} \mathrm{~nm}$ sized Au structures can be catalytically active. ${ }^{27-34}$ Here we show that even on well-ordered $\mathrm{Au}(111)$ surfaces, some functionalized trioxatriangulenium (TOTA) platform molecules fragment despite the fact that they physisorb. ${ }^{35,36}$ We functionalized TOTA platforms with methyl, ethyl, ethynyl, propynyl, and hydrogen. After sublimation onto $\mathrm{Au}(111)$, all methylTOTA and $>99 \%$ ethyl-TOTA on Au(111) are intact. However, $\approx 60 \%$ of the H-TOTA molecules and almost all (>99\%)

a)Electronic mail: jasper-toennies@physik.uni-kiel.de
propynyl-TOTA and ethynyl-TOTA decompose. Intriguingly, this tendency toward fragmentation on $\mathrm{Au}(111)$ is opposite to the sequence of gas-phase stabilities of the molecules. Density functional theory (DFT) calculations show that the different on-surface stabilities reflect the binding energies of the products to $\mathrm{Au}(111)$. Dispersion interaction binds the platform molecules to $\mathrm{Au}(111)$ and affects the positions of the intact molecules and the decomposition products but hardly affects the stability against fragmentation.

\section{EXPERIMENTAL RESULTS}

\section{A. Deposition of TOTA functionalized with methyl, $\mathrm{H}$, and ethynyl onto $\mathrm{Au}(111)$}

Functionalized platforms 1-3 (Fig. 1) were sublimated onto $\mathrm{Au}(111)$ at mild parameters to make sure that intact molecules were deposited (see the Appendix). During sublimation, the samples were held at ambient temperature and then investigated using scanning tunneling microscope (STM) at low temperature $(T=4.6 \mathrm{~K})$.

Figures 1(a) and 1(b) present typical STM topographs recorded after sublimation of molecule 1. Pristine Au(111) areas appear low (dark). A single molecule $\mathbf{1}$ is imaged as a triangular shape with a central circular protrusion [green bordered molecule in Fig. 1(b)]. The triangle (height $\lesssim 0.16 \mathrm{~nm}$ ) and the central protrusion $(0.23 \mathrm{~nm})$ correspond to platform 1 and the attached $\mathrm{CH}_{3}$ moiety, respectively. The platform lies flat on $\mathrm{Au}(111)$ with an upright $\mathrm{CH}_{3}$ group as desired. The molecules arrange into hexagonal clusters, preferably on the hcp and fcc areas of the herring-bone reconstructed $\mathrm{Au}(111)$ substrate. These clusters are stabilized by $\mathrm{CH} \cdots \mathrm{O}$ bonds with bond angles of $150^{\circ}-170^{\circ}$ and $\mathrm{H}-\mathrm{O}$ distances between 0.27 and $0.32 \mathrm{~nm}$. Although we imaged thousands of molecules, 

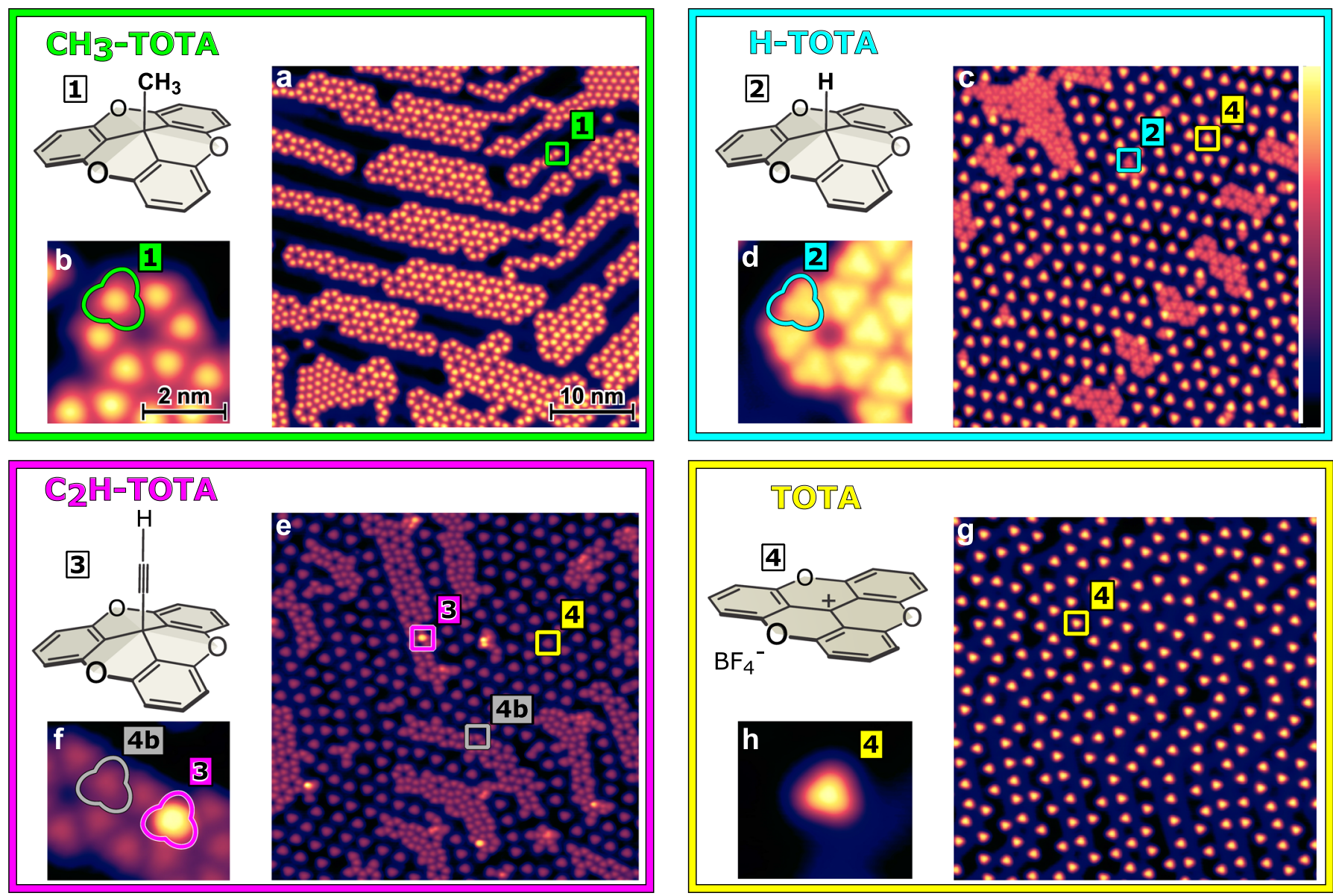

FIG. 1. [(1)-(3)] Functionalized trioxatriangulenium (TOTA) platform molecules: methyl-TOTA (1), H-TOTA (2), and ethynyl-TOTA (3) molecules. (4) Bare TOTA platform (4) with counterion $\mathrm{BF}_{4}^{-}$. [(a), (c), (e), and (g)] STM topographs of (1-4), respectively, on Au(111). Image width $44 \mathrm{~nm}$. [(b), (d), (f), and (h)] close-up views, image width $4.4 \mathrm{~nm}$, of (1-4). green, cyan, magenta, yellow, and gray symbols highlight single molecules (1-4) and (4b), respectively. The color scale shown in (c) is used throughout but corresponds to different maximum height in each image. (1-4) and (4b) exhibit maximum apparent heights of 0.23 , $0.16,0.36,0.22$, and $0.21 \mathrm{~nm}$ at $100 \mathrm{mV}$. (e) was recorded at $-1.0 \mathrm{~V}$.

we did not find a single fragmented molecule. In other words, molecule 1 remains intact on $\mathrm{Au}(111)$.

The deposition of molecule 2 leads to a different result [Figs. 1(c) and 1(d)]. Here two patterns with apparent heights of 0.16 and $0.21 \mathrm{~nm}$ [cyan and yellow squares in Figs. 1(c) and 1(d)] were found. The higher pattern (yellow) is identical to the pattern found after deposition of the bare platform 4 [Figs. 1(g) and 1(h)]. Consequently, the higher pattern is attributed to 4. In Fig. 1(c), 4 is a fragment of molecule 2. Its occurrence indicates that the bond between the central $\mathrm{H}$ atom, and the platform occasionally dissociates upon deposition. The lower pattern [cyan in Figs. 1(c) and 1(d)] is attributed to the intact molecule 2 . In drastic contrast to the methyl-functionalized molecule 1 , only $\approx 41 \%$ of more than 3000 molecules 2 were intact after deposition. The central $\mathrm{H}$ atom of molecule $\mathbf{2}$ is not individually resolved as expected (see, e.g., Refs. 37 and 38). Despite its similar geometry, molecule 2 appears $\approx 0.06 \mathrm{~nm}$ lower than the bare platform $\mathbf{1}$. This difference is caused by an electronic effect as discussed below.

Figures 1(e) and 1(f) present topographs recorded after deposition of molecules $3\left(\mathrm{C}_{2} \mathrm{H}-\mathrm{TOTA}\right)$. Again, the bare platform 4 [yellow square in Fig. 1(e)] is found as a fragment on the sample. In addition, a different pattern denoted 4b [gray markers in Figs. 1(e) and 1(f)] occurs. The pattern $\mathbf{4 b}$ is exclusively found after deposition of $\mathbf{3}$ with fractions varying between $5 \%$ and $80 \%$ at coverages of 0.05-0.45 molecules per $1 \mathrm{~nm}^{2}$. As discussed below, we attribute the pattern $\mathbf{4 b}$ to the bare platform in a different adsorption state than $\mathbf{4}$. Intact molecules 3 [magenta in Figs. 1(e) and 1(f)] appear highest $(0.36 \mathrm{~nm})$ because of the attached ethynyl group. However, less than $1 \%$ of $\mathbf{3}$ are intact on $\mathrm{Au}$, showing that the $\mathrm{C}_{2} \mathrm{H}$ moiety makes these molecules marginally stable on $\mathrm{Au}(111)$.

\section{B. Intermolecular interactions}

The topographs in Figs. 1(c), 1(e), and 1(g) provide important additional information. In contrast to the functionalized molecules 1-3 and molecule $\mathbf{4 b}$, the bare platform 4 does not cluster. The molecules 4 rather prefer to maximize their average nearest-neighbor distance $d$ in fcc and hcp regions of the substrate. The square of the average nearest-neighbor distance, $d^{2}$, describes (up to a constant factor) the surface 
area that is occupied by a single molecule. Given a long-range repulsive interaction, $d^{2}$ is expected to be inversely proportional to the coverage, which determines the area that is available for a single molecule. Figure 2(a) shows that this proportionality indeed holds. Moreover, it evidences that $d$ is not limited to specific values. This observation excludes the possibility of surface-mediated long-range interactions via Friedel oscillations of surface-state electrons, where $d$ would change with a period of half of the Fermi wavelength, i.e., $1.8 \mathrm{~nm}$ for $\mathrm{Au}(111){ }^{39-41}$

Long-range interactions between adsorbates can also be mediated by elastic deformation of the substrate. ${ }^{42-44}$ The herring-bone reconstruction, which is due to a stressed atomic layer, is sensitive to changes of the surface elastic energy. ${ }^{45,46}$ However, we find that the herring-bone reconstruction is unaffected by submonolayer coverages of molecule 4. Moreover, the platform is physisorbed to the Au substrate and consequently the induction of a significant amount of strain is unlikely. Hence, an elastic surface-mediated interaction is improbable.

Next, the nearest-neighbor (NN) pair distribution functions for low coverages were investigated [Figs. 2(b) and 2(c)]. They show that the NN distances have lower and upper limits, e.g., in case of Fig. 2(b), there are no NN distances $<2$ or $>3.5 \mathrm{~nm}$. This effect along with the shape of the distributions indicates long-range Coulomb repulsion between the molecules ${ }^{46}$ and suggests a significant charging of the adsorbed platform $\mathbf{4}$, consistent with its cationic nature in the gas phase.

Clusters of molecules 1-3 and 4b [Figs. 1(a), 1(c), and 1(e)] exhibit the same hexagonal structure and orientation with respect to the substrate. They are stabilized by $\mathrm{CH} \cdots \mathrm{O}$ bonds between the platforms. The formation of $\mathrm{H}$ bonds indicates a smaller intermolecular Coulomb repulsion and consequently less charging of the molecules, consistent with their neutral character in the gas phase. In fact, the on-surface interactions of 1-4 reflect the molecular tendency to ionize. A related switching of intermolecular interactions by attaching a ligand to a molecule has recently been reported for Fe-tetramethyl-tetraazaannulene on $\mathrm{Au}(111) .{ }^{47}$

\section{Induced dissociation and electronic states near $E_{F}$}

As presented above, methyl-TOTA (1) is stable on $\mathrm{Au}(111)$ at ambient temperature, H-TOTA (2) fragments, and ethynyl-TOTA (4) is marginally stable. Moreover, the occurrence of the bare platform as fragment indicates that the bond between platform and attached moiety dissociates upon deposition. To verify that this bond is weaker than the other bonds of the adsorbed molecules, we investigated the stability of the molecules upon injecting high currents into the molecules. Indeed, injection of several $\mathrm{nA}$ current into the molecules 1-3 at elevated voltages ( $\gtrsim 2 \mathrm{~V}$ ) dissociates the attached moieties from the platform but does not lead to further fragmentation of the platform. An example for such an induced dissociation is shown in Fig. 3 for molecule $\mathbf{1}$ on $\mathrm{Au}(111)$. Initially [Fig. 3(a)], methyl-TOTA molecules arranged in a compact island are observed. After applying a current/voltage pulse to a molecule $\mathbf{1}$ at the position indicated by a black point, a new state is obtained [Fig. 3(b)] whose characteristics match the bare platform 4. This kind of dissociation, which was repeatedly observed, is selective, i.e., no other molecules are affected. After an induced dissociation, the STM tip was usually contaminated, i.e., contacts to the bare $\mathrm{Au}$ substrate revealed a non-metallic tip and STM images tended to be fuzzy. We attribute this contamination to the transfer of the functional group or fragments thereof to the tip. After tip preparation by repeated dipping into the Au surface,
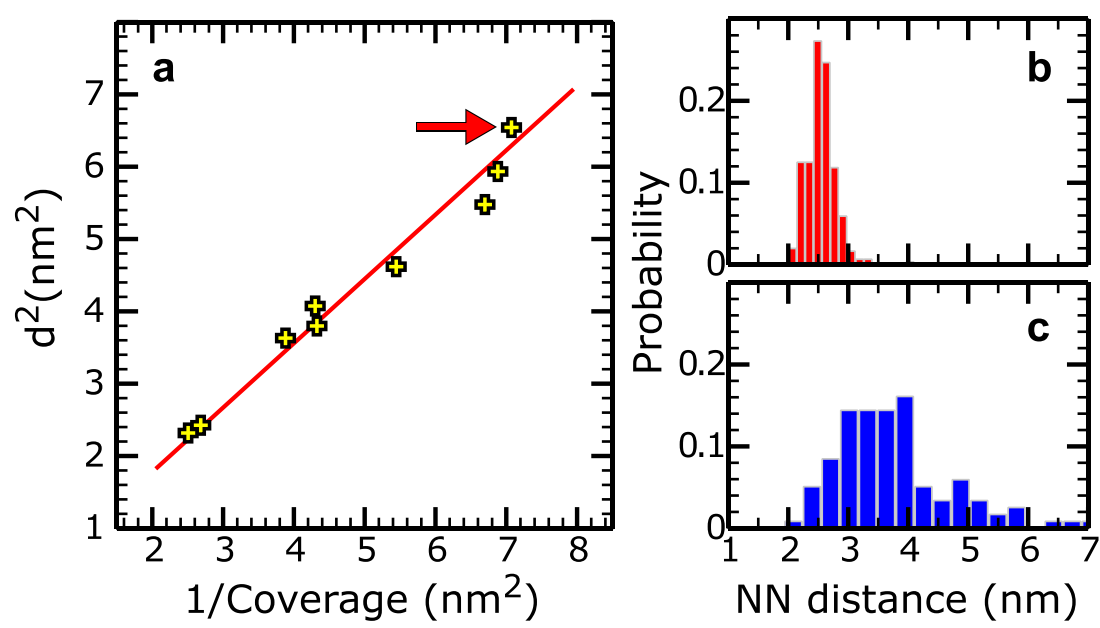

FIG. 2. (a) Average nearest-neighbor distance squared $\left(d^{2}\right)$ versus inverse coverage evaluated for 4 . Each data point is an average over at least 300 molecules from large areas of a newly prepared sample. This average neglects differences in local coverage between hcp and fcc areas that are significant at low densities ( $\gtrsim 7 \mathrm{~nm}^{2}$ per molecule). The line shows a linear fit to the data $\left(d^{2}=a / \gamma, \gamma\right.$ being the coverage in molecules per nm ${ }^{2}$ ). We find $a=0.89$, which is close to the value expected for a hexagonal lattice $(\sqrt{3} / 2 \approx 0.87)$, which the molecules approximately form [cf. molecules 4 in Figs. 1(c) and 1 (e)]. [(b)-(c)] Nearestneighbor (NN) pair distribution functions of $\mathbf{4}$ for coverages (b) 0.14 and (c) 0.03 molecules per nm ${ }^{2}$ [Figs. 1 (g) and $4(\mathrm{a})$, respectively]. The distributions were obtained from $>200$ molecules. Steric hindrance limits the smallest distance to $\approx 1 \mathrm{~nm}$. The data point marked by an arrow in (a) corresponds to the data shown in (b). 

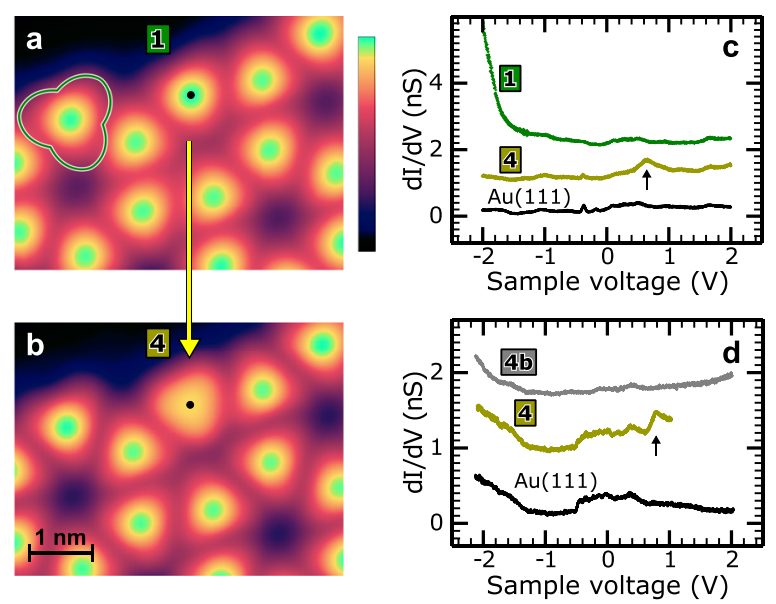

FIG. 3. [(a) and (b)] STM topographs (a) before and (b) after STM-induced dissociation of the methyl moiety from the single molecule $\mathbf{1}$ indicated by black points. [(c) and (d)] Differential conductance ( $d I / d V)$ spectra of molecule 1, 4, and $\mathbf{4 b}$ along with the respective background spectra of the bare $\mathrm{Au}(111)$ substrate. Black arrows indicate the positions of the LUMO peak of $\mathbf{4}$ when it is (c) isolated or (d) adjacent to $\mathbf{1}$. The steep rise of the conductance of $\mathbf{1}$ at $\approx-1.8 \mathrm{eV}$ is attributed to the HOMO. The Au(111) spectrum in (d) exhibits a rapid rise at $\approx-0.5 \mathrm{~V}$ that is related to the edge of the surface state. The increase of the $d I / d V$ signal in (d) for sample voltages $\lesssim-1.5 \mathrm{eV}$ is due to tip states. For clarity, some spectra are vertically shifted: (c) $\mathbf{1}:+1.5 \mathrm{nS}$ and $\mathbf{4}:+0.9 \mathrm{nS}$ and (d) $\mathbf{4 b}:+0.9 \mathrm{nS}$ and $\mathbf{4}$ : $+0.3 \mathrm{nS}$. The feedback loop was opened at $0.5 \mathrm{nA}$ and $2 \mathrm{~V}[1 \mathrm{~V}$ for 4 in (d)].

STM images could again be recorded with a clean metal tip. Figure 3(b) was actually recorded with such a re-prepared tip.

Further information about the electronic states of adsorbates can be obtained with the help of differential conductance $(d I / d V)$ spectra, which probe the local density of states (LDOS). $d I / d V$ spectra of 1 and $\mathbf{4}$ [Fig. 3(c)] reveal a LUMO peak at $\approx 0.6 \mathrm{eV}$ for the bare platform 4 that is not present at molecule $\mathbf{1}$. Actually all investigated functionalized platforms do not exhibit any prominent state in the range $\left|E-E_{F}\right| \lesssim 1.5 \mathrm{eV}$. For molecule 1, a steep increase of the differential conductance is visible for energies below $E-E_{F} \lesssim-1.5 \mathrm{eV}$. This is an onset of a HOMO peak. A complete measurement of this peak at the tunneling parameters of Fig. 3(c) would lead to the aforementioned induced dissociation of 3. The $d I / d V$ spectra of $\mathbf{4}$ in Figs. 3(c) and 3(d) reveal a further piece of information. The energy of the LUMO of 4 depends on its environment. Isolated molecules 4 show a LUMO at $\approx 0.6 \mathrm{eV}$ [Fig. 3(c)]. An adjacent functionalized platform shifts the level away from $E_{F}$ by $\approx 0.2 \mathrm{eV}$ [Fig. 3(d)]. Polarization screening of neighboring molecules may cause this shift as observed for, e.g., perylenetetracarboxylic-dianhydride on $\mathrm{Au}(111)^{48}$ and tetracene on $\operatorname{Ag}(111) .49$

Besides the bare platform $\mathbf{4}$, also $\mathbf{4 b}$ was identified as a fragment of $\mathbf{3}$ [Figs. 1(e) and 1(f)]. The $d I / d V$ spectra of $\mathbf{4}$ and $\mathbf{4 b}$ [Fig. 3(d)] reveal a marked difference of their electronic structures. Whereas 4 exhibits a LUMO resonance at below $1 \mathrm{eV}$, fragment $\mathbf{4 b}$ has no state for $\left|E-E_{F}\right| \lesssim 2 \mathrm{eV}$. The absence of an unoccupied state close to $E_{F}$ along with the clustering of 4b (see Sec. II B) is presumably caused by a different charge state.

\section{Decomposition products}

As to fragment $\mathbf{4 b}$, several observations indicate that it is the bare platform in a different adsorption state on $\mathrm{Au}(111) . \mathbf{4 b}$ is found exclusively after decomposition of $\mathbf{3}$ [Figs. 1(e) and 1(f)]. Neither the sublimation of the bare platform [Figs. 1(g) and 1(h)] nor the decomposition of 2 [Figs. 1(c) and 1(d)] lead to the presence of $\mathbf{4 b}$. Thus, its formation is most likely a kinetic effect of the dissociation of 3 . As an intermediate, molecule $\mathbf{4 b}$ should be energetically less favorable than $\mathbf{4}$. Consistent with this, $\mathbf{4 b}$ can be converted to $\mathbf{4}$ by injecting current into the molecules at elevated voltages $(\gtrsim 2 \mathrm{~V})$ and the reverse process could not be achieved despite various attempts at different manipulation parameters. Another intriguing observation is that the fraction of $\mathbf{4 b}$ after deposition of $\mathbf{3}$ drastically depends on the coverage of the molecules [Figs. 4(a) and 4(b)]. Low (high) coverages correlate with small (high) fractions of molecule $4 \mathbf{b}$ [e.g., $0.05(0.45)$ molecules per $1 \mathrm{~nm}^{2}$ translates to $\approx 5 \%(80 \%) 4 \mathbf{b}]$. When the coverage is increased from 0.29 [Fig. 1(e)] to 0.45 molecules per $1 \mathrm{~nm}^{2}$ [Fig. 4(b)], the intermolecular distance of the bare platform slightly reduces by $\approx 0.1 \mathrm{~nm}$. However, the fraction of $\mathbf{4 b}$ increases from $59 \%$
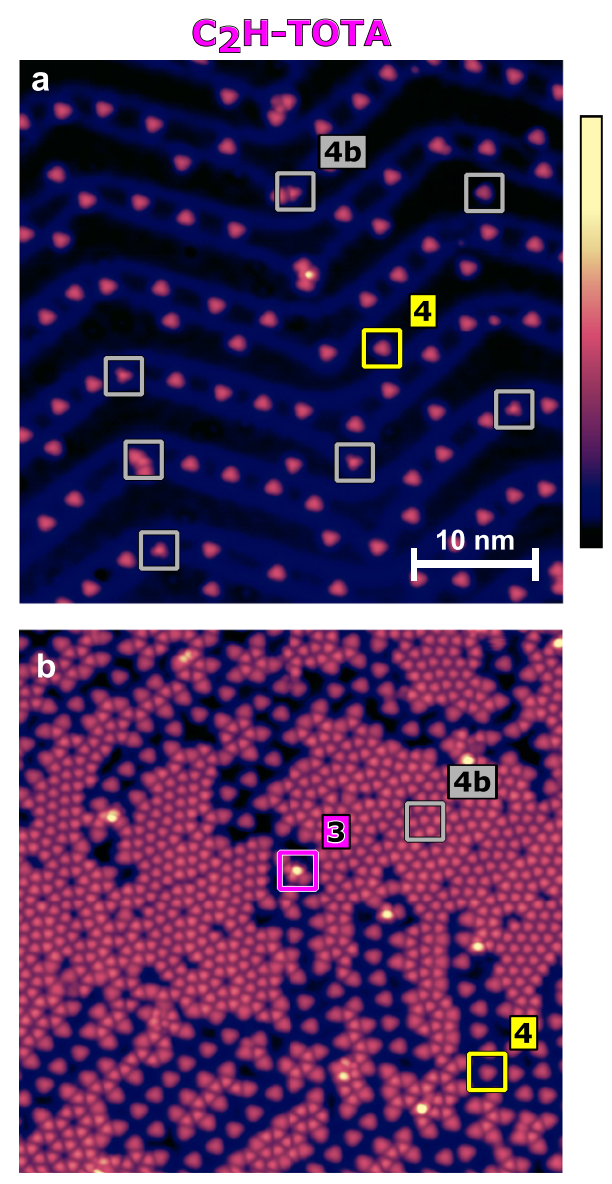

FIG. 4. STM topographs after deposition of different coverages of molecule 3 on Au(111): (a) 0.05 and (b) 0.45 molecules per $1 \mathrm{~nm}^{2}$ (image width $44 \mathrm{~nm}$ ). In (a), the fragments $\mathbf{4 b}$ are at the reactive elbows of the herring-bone reconstruction and marked by a gray square, respectively. In (b), the molecules $\mathbf{4 b}$ (gray) cluster to large islands. Molecules $\mathbf{4}$ (yellow) do not cluster. The color scale shown in (a) is used and corresponds to an apparent height of $3.7 \mathrm{~nm}$. (b) was recorded at $-1 \mathrm{~V}$. 
to $80 \%$, i.e., most of the additional molecules disintegrate into molecule $\mathbf{4 b}$. This coverage dependence indicates that the interaction with other molecules hinders the conversion of 4b to $\mathbf{4}$. Since $\mathbf{4}$ is significantly charged (see Sec. II B), this hindrance may be linked to the intermolecular Coulomb repulsion of $\mathbf{4}$ that renders $\mathbf{4}$, in contrast to $\mathbf{4 b}$, energetically less favorable at higher coverages.

The aforementioned properties of $\mathbf{4 b}$ are all similar to those of the functionalized platforms and could be explained by a moiety attached to the platform. However, there were, in contrast to the induced dissociations of the functionalized platforms (see Sec. II C), no indications of a detached moiety after conversion of $\mathbf{4 b}$ to $\mathbf{4}$. As molecule $\mathbf{4 b}$ is a fragment of $\mathrm{C}_{2} \mathrm{H}$-TOTA, the attached moiety should comprise either one or two $\mathrm{C}$ atoms. Such moieties are neither chemically intuitive [high gas-phase dissociation energies of the related bonds: (i) $\mathrm{HC}_{2}-\mathrm{H}: 5.8 \mathrm{eV}(560 \mathrm{~kJ} / \mathrm{mol})$ and (ii) $\mathrm{HC} \equiv \mathrm{CH}: 10 \mathrm{eV}$ $(965 \mathrm{~kJ} / \mathrm{mol})]$ nor consistent with the STM topographs. Hence, fragment $\mathbf{4 b}$ is most likely the bare platform in a metastable adsorption state.

While fragments $\mathbf{4}$ and $\mathbf{4 b}$ are directly observed upon dissociation of 3 [Figs. 1(e) and 1(f)], the fate of the $\mathrm{C}_{2} \mathrm{H}$ group is less clear. STM topographs reveal fuzzy areas (supplementary material, Sec. II) that may represent clusters of $\mathrm{C}_{2} \mathrm{H}$. The high reactivity of $\mathrm{C}_{2} \mathrm{H}$ may also lead to the formation of gaseous molecules like $\mathrm{HC}_{2}-\mathrm{C}_{2} \mathrm{H}$ [bond formation energy $-6.5 \mathrm{eV}$ $(627 \mathrm{~kJ} / \mathrm{mol})$ in the gas phase $]^{50}$ and their partial desorption at ambient temperature.

The fragmentation of molecule 2 upon adsorption on $\mathrm{Au}(111)$ at ambient temperature [Fig. 1(c)] may lead to $\mathrm{H}$ atoms on the sample-in addition to the bare platforms 4 . $\mathrm{H}$ atoms on $\mathrm{Au}(111)$ were reported to desorb at $\approx 110 \mathrm{~K}$ via the formation of $\mathrm{H}_{2} \cdot{ }^{51} \mathrm{In}$ agreement with that, we found no indications of their presence on our samples.

\section{CALCULATED RESULTS}

The $\mathrm{CH}_{3}$ and $\mathrm{C}_{2} \mathrm{H}$-functionalized platforms $\mathbf{1}$ and $\mathbf{3}$ are extreme cases with either exclusively intact molecules or $>99 \%$ fragments on $\mathrm{Au}(111)$. We therefore calculated their dissociation energies with DFT assuming different reaction scenarios (see below). Moreover, we focused on the bare platform 4 as a product because we hint that $4 \mathrm{~b}$ may result from a cooperative effect that is difficult to tackle with existing methods. In other words, our calculations reflect the case of small coverages where most of the deposited molecules $\mathbf{3}$ fragment to 4 . To adequately describe the adsorption geometries, dispersion interactions were taken into account by using the $\mathrm{vdW}^{\text {surf }}$ method (see the Appendix). ${ }^{52-54}$

\section{A. Dissociation energies}

Reaction I represents the gas-phase dissociations of molecules $\mathbf{1}$ and $\mathbf{3}$ (Fig. 5). It turns out (Table I) that $\mathbf{1}$ and $\mathbf{3}$ are stable with dissociation energies exceeding $2 \mathrm{eV}$ $(190 \mathrm{~kJ} / \mathrm{mol})$, which is consistent with the observed stability toward sublimation. Moreover, in contrast to the observed instability on $\mathrm{Au}(111)$, the bond of the functional
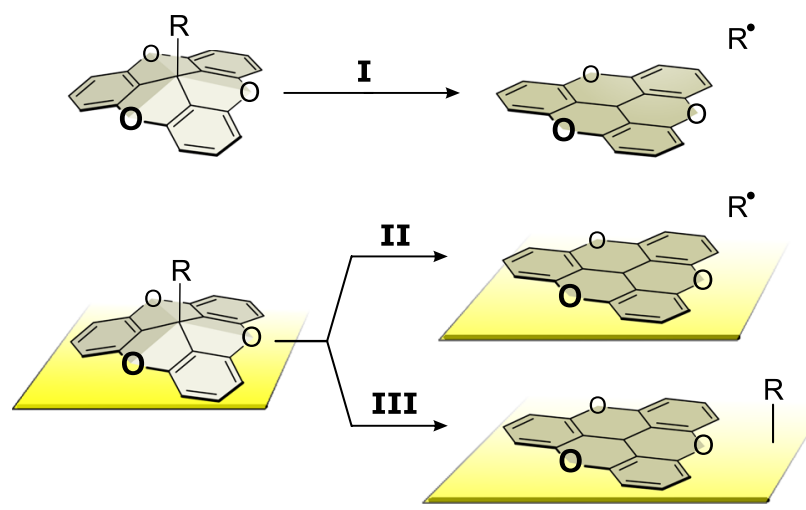

FIG. 5. Sketch of gas-phase dissociation I and on-surface dissociations II and III of the substituent R from the platform. In the on-surface dissociations, $\mathrm{R}$ either (II) desorbs or (III) adsorbs to $\mathrm{Au}(111)$.

group is $1 \mathrm{eV}$ stronger in $\mathbf{3}\left(\mathrm{C}_{2} \mathrm{H}-\mathrm{TOTA}\right)$ than in $\mathbf{1}\left(\mathrm{CH}_{3}-\right.$ TOTA). Reaction $\mathbf{I}$ therefore cannot explain the experimental observations.

Reaction II describes the on-surface dissociation assuming that the platform remains on $\mathrm{Au}$ and the functional group desorbs (Fig. 5). Interestingly, both dissociation energies (II in Table I) are $\approx 0.45 \mathrm{eV}$ (43 kJ/mol) lower than the gas-phase values. This destabilization is mainly caused by the more favorable adsorption of the flat platform $4(\approx 0.4 \mathrm{eV})$ compared to the curved platforms with the attached functional group (Fig. 5). It is not due to the geometric deformation caused by the adsorption. Since both dissociation energies exceed $1.5 \mathrm{eV}$ (144 kJ/mol) and $\mathbf{3}$ is $1 \mathrm{eV}(96 \mathrm{~kJ} / \mathrm{mol})$ more stable than $\mathbf{1}$, II does not reflect the experimental observations.

Reaction III is an on-surface dissociation with both products remaining on Au (Fig. 5). Remarkably, III reveals that $\mathbf{3}$ $\left(\mathrm{C}_{2} \mathrm{H}-\mathrm{TOTA}\right)$ is not stable on $\mathrm{Au}$, while $\mathbf{1}\left(\mathrm{CH}_{3}-\mathrm{TOTA}\right)$ is stable (Table I). The dissociation of $\mathbf{3}$ reduces the total energy by $\approx 0.1 \mathrm{eV}(9.6 \mathrm{~kJ} / \mathrm{mol})$, whereas dissociation of $\mathbf{1}$ requires more than $0.4 \mathrm{eV}(38 \mathrm{~kJ} / \mathrm{mol})$. In other words, the on-surface dissociation III is consistent with the experimental observation of mainly decomposed $\mathbf{3}$ and fully intact $\mathbf{1}$ on Au.

TABLE I. Dissociation energies of $\mathbf{1}\left(\mathrm{CH}_{3}-\right.$ TOTA $)$ and $\mathbf{3}\left(\mathrm{C}_{2} \mathrm{H}-\mathrm{TOTA}\right)$ in $\mathrm{eV}(96 \mathrm{~kJ} / \mathrm{mol})$ for calculated reaction pathways I-III. The dissociation energies were obtained as described in the section DFT of the Appendix.

\begin{tabular}{llrr}
\hline \hline & \multicolumn{1}{c}{ Dissociation } & $\mathbf{1}$ & \multicolumn{1}{c}{$\mathbf{3}$} \\
\hline I & $\mathrm{R}-$ TOTA $\rightarrow \mathrm{R}+$ TOTA & 2.43 & 3.77 \\
II & $\mathrm{R}-$ TOTA $/ \mathrm{Au} \rightarrow \mathrm{R}+$ TOTA/Au & 1.98 & 3.30 \\
III & $\mathrm{R}-$ TOTA $/ \mathrm{Au} \rightarrow \mathrm{R} / \mathrm{Au}+$ TOTA/Au & 0.46 & -0.10 \\
\hline \hline
\end{tabular}

TABLE II. Adsorption energy in $\mathrm{eV}(96 \mathrm{~kJ} / \mathrm{mol})$ of the molecules $\mathbf{1}, \mathbf{3}, \mathbf{4}$ and the $\mathrm{CH}_{3}$ and $\mathrm{C}_{2} \mathrm{H}$ radicals on $\mathrm{Au}(111)$. The adsorption energies were obtained as described in the section $D F T$ of the Appendix.

\begin{tabular}{lcccrr}
\hline \hline & $\mathbf{1}^{\mathrm{a}}$ & $\mathbf{3}^{\mathrm{b}}$ & $\mathbf{4}^{\mathrm{c}}$ & $\mathrm{CH}_{3}$ & $\mathrm{C}_{2} \mathrm{H}$ \\
\hline$E_{a d}(\mathrm{PBE}+\mathrm{vdW})$ & -2.17 & -2.15 & -2.62 & -1.5 & -3.4 \\
$E_{a d}(\mathrm{PBE})$ & +0.01 & -0.02 & -0.57 & -1.3 & -3.2 \\
\hline \hline
\end{tabular}

${ }^{\mathrm{a}} \mathrm{CH}_{3}-$ TOTA.

${ }^{\mathrm{b}} \mathrm{C}_{2} \mathrm{H}-\mathrm{TOTA}$.

${ }^{\mathrm{c}}$ TOTA. 

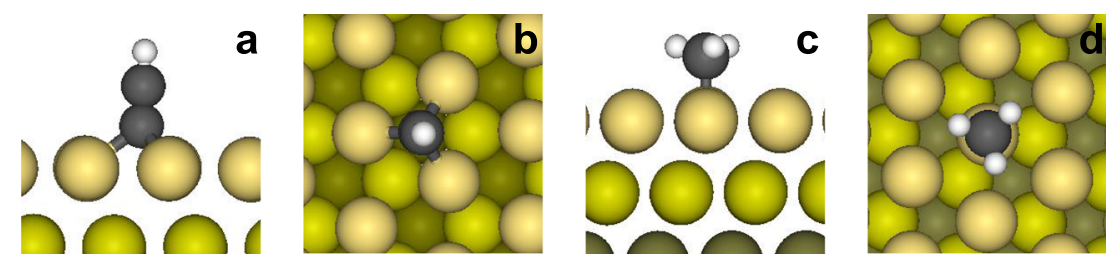

FIG. 6. Adsorption geometries of [(a) and (b)] $\mathrm{C}_{2} \mathrm{H}$ and [(c) and (d)] $\mathrm{CH}_{3}$ calculated with PBE + vdW (see the Appendix). (a) and (c) show side views and (b) and (d) show top views. $\mathrm{C}_{2} \mathrm{H}$ and $\mathrm{CH}_{3}$ are adsorbed in hollow and top sites, respectively. Yellowish: Au atoms of the first three (111) surface layers, gray: carbon, and white: hydrogen.

In addition to the dissociation pathways I-III, it is conceivable that the $\mathrm{R}$ moieties of two R-TOTA molecules directly react to $\mathrm{R}-\mathrm{R}$ without the adsorption of the $\mathrm{R}$ moieties on the substrate. In other words, the reaction $2 \mathrm{R}-\mathrm{TOTA} / \mathrm{Au} \rightarrow \mathrm{R}-\mathrm{R}+2$ TOTA/Au (denoted as IV below) might occur without reaction III as an intermediate step. However, the dissociation energies of reaction $\mathbf{I V}, 0.10$ and $0.06 \mathrm{eV}$ for $\mathrm{R}$ equal to $\mathrm{C}_{2} \mathrm{H}_{2}$ and $\mathrm{CH}_{3}$, respectively, are almost identical. As a consequence, reaction IV (without reaction III as an intermediate step) cannot explain the drastically different stabilities of molecules $\mathrm{C}_{2} \mathrm{H}_{2}$-TOTA (almost all dissociated) and $\mathrm{CH}_{3}$-TOTA (all intact) on $\mathrm{Au}(111)$, in contrast to reaction III.

\section{B. Adsorption energies and geometries}

The key reason for the different stabilities on $\mathrm{Au}(111)$ are the adsorption energies of $\mathrm{C}_{2} \mathrm{H}$ and $\mathrm{CH}_{3}$ (Table II). The former is $1.9 \mathrm{eV}(180 \mathrm{~kJ} / \mathrm{mol})$ more favorable than that of $\mathrm{CH}_{3}$. Along with the lower adsorption energy of the bare platform 4 , this energy difference is enough to render $\mathbf{3}$ unstable whereas $\mathbf{1}$ is not. Hence, the $\mathrm{Au}(111)$ surface is not inert enough for $\mathbf{3}$ to remain stable. Consistent with this interpretation, we observed that 3 dissociates on the more reactive $\operatorname{Ag}(111)$ surface, too (supplementary material, Sec. III).

Figure 6 shows the optimized geometries of $\mathrm{C}_{2} \mathrm{H}$ and $\mathrm{CH}_{3}$ on $\mathrm{Au}(111) . \mathrm{C}_{2} \mathrm{H}$ adsorbs upright at fcc-hollow sites [Figs. 6(a) and 6(b)], whereas $\mathrm{CH}_{3}$ is located on top of a surface $\mathrm{Au}$ atom [Figs. 6(c) and 6(d)]. The calculated Au-C distances, $0.221 \mathrm{~nm}$ for $\mathrm{C}_{2} \mathrm{H}$ and $0.212 \mathrm{~nm}$ for $\mathrm{CH}_{3}$, are typical of covalent $\mathrm{Au}-$ $\mathrm{C}$ bonds. ${ }^{55}$ The strong binding of $\mathrm{C}_{2} \mathrm{H}$ reflects the fact that $\mathrm{C}_{2} \mathrm{H}$ binds to three $\mathrm{Au}$ atoms rather than one in the case of $\mathrm{CH}_{3}$. Interestingly, $\mathrm{CH}_{3}$ is marginally stable or unstable at the hollow sites, as indicated by the relaxation to the top site in the DFT calculations. We attribute this effect to the close proximity of the $\mathrm{H}$ atoms in $\mathrm{CH}_{3}$ and the $\mathrm{Au}(111)$ for the expected $\mathrm{C}-\mathrm{Au}$ bond distance of $0.21 \mathrm{~nm}$ at the hollow sites. Details of the calculated adsorption geometries of the platforms $\mathbf{1}, \mathbf{3}$, and $\mathbf{4}$ are presented in the supplementary material (Sec. VII).

\section{Role of dispersion interaction}

The decomposition of several molecules and the relative stabilities of intermediates in on-surface reactions on $\mathrm{Au}$ have recently been attributed to the strong van der Waals $(\mathrm{vdW})$ interaction. ${ }^{56-60}$ We therefore investigated the role of dispersion for dissociation II and III by calculating the related adsorption energies with and without inclusion of
$\mathrm{vdW}$ interaction (Table II), namely, with the PBE + vdW functional and the pure PBE (Perdew-Burke-Ernzerhof) functional (see Appendix, Subsection 4). The adsorption energies of $\mathbf{1}, \mathbf{3}$, and $\mathbf{4}$ increase by $\approx 2.2 \mathrm{eV}$ with PBE compared to $\mathrm{PBE}+\mathrm{vdW}$, reflecting strong dispersion interaction with $\mathrm{Au}(111)$ and its crucial role for the adsorption process. The adsorption energies of $\mathbf{1}$ and $\mathbf{3}$ may contribute to overcoming the energy barrier of dissociation process III. However, the calculated adsorption energies of $\mathbf{1}$ and $\mathbf{3}$ are almost identical and therefore cannot explain the observed stability difference. Similarly, dispersion interaction has little influence on the dissociation energies when the correct adsorption geometries are used. The energy differences relevant for dissociation paths II and III, namely, between $\mathbf{4}$ on one hand and $\mathbf{1}$ and $\mathbf{3}$ on the other $(\approx 0.4 \mathrm{eV})$ and between $\mathrm{CH}_{3}$ and $\mathrm{C}_{2} \mathrm{H}(\approx 1.9 \mathrm{eV})$, are almost unaffected when changing from $\mathrm{PBE}+\mathrm{vdW}$ to PBE. Hence, the dispersion interaction plays an indirect role in the reactions. It defines the position of the adsorbates on the $\mathrm{Au}(111)$ surface but cancels out from the reaction energies. We emphasize that $\mathrm{vdW}$ interactions are crucial as not taking them into account, i.e., only using the pure PBE functional, leads to incorrect adsorption geometries and unreasonable dissociation energies.

\section{DISCUSSION}

Experimentally we observed that molecule $\mathbf{3}$ fragments with a very high probability. If a reaction barrier is present, it obviously can be overcome at ambient temperature. In any event, according to our DFT results, dissociation lowers the energy of the system. As to molecule $\mathbf{1}$, our calculations show that dissociation is energetically unfavorable in agreement with its observed stability. An activation barrier toward dissociation may add to this stability but cannot be evaluated from the available data.

The pathway to dissociation is a multi-step process, and it is not realistic to model the complete dissociation with existing $a b$ initio methods. ${ }^{61}$ Nonetheless, it is interesting to speculate about the mechanisms of fragmentation and molecular assembly. Dissociation III may require that the functional group is oriented toward the surface. Disregarding a hypothetical steering effect of the surface during the impact of the molecules, approximately half of the molecules are expected to impinge with the platform oriented toward the surface. The observations for 3 [Fig. 1(e)] imply that this orientation during impact is no obstacle to fragmentation. However, the barrier for flipping 3 on a flat area is expected to exceed $1 \mathrm{eV}(96 \mathrm{~kJ} / \mathrm{mol})$ because of its large adsorption energy (Table II). Diffusion of 
the molecules to step edges may facilitate a reorientation. It is also conceivable that diffusing Au atoms may attack the ligand bond of 3 .

Our interpretation of the fragmentation data is consistent with the results from other functionalized TOTA platforms. We synthesized propynyl-TOTA, which may be viewed as methylTOTA with an inserted ethynyl spacer. As expected, we find propynyl-TOTA to be only marginally stable on $\mathrm{Au}(111)$ with $<1 \%$ molecules remaining intact (see supplementary material, Sec. IV). The stability of ethyl-TOTA is also in line with our model. The gas-phase dissociation energy of ethyl-TOTA and the binding energy of ethyl to $\mathrm{Au}(111)$ are expected to be similar to those of methyl. Indeed, in our experiments, we found almost only (>99\%) intact ethyl-TOTA molecules on $\mathrm{Au}(111)$ (see supplementary material, Sec. IV).

\section{CONCLUSION}

The interaction of closed-shell platform molecules with the inert $\mathrm{Au}(111)$ surface surprisingly renders a rather stable $s p_{3} \mathrm{C}$ bond within a molecule unstable. The stability of the molecules depends drastically on their functional moiety. Modeling shows that the binding energies of the fragments to $\mathrm{Au}(111)$ destabilize the $s p_{3} \mathrm{C}$ bond between the functional moieties and the platform by $2-3.9 \mathrm{eV}(190-370 \mathrm{~kJ} / \mathrm{mol})$ and essentially determine the molecular stability. Dispersion interaction plays an important indirect role, as it binds the platforms to the $\mathrm{Au}(111)$ surface, but it cancels out from the reaction energies. Moreover, the results show that the thermal energy at ambient temperature and the impact of the molecules are sufficient to overcome kinetic limitations.

\section{SUPPLEMENTARY MATERIAL}

See Supplementary material for details of synthesis and calculations; calculated MODOS of adsorbed molecules $\mathbf{1}$ and 4; topographs of propynyl-TOTA, ethyl-TOTA, and ethynyl fragments on $\mathrm{Au}(111)$; topographs of $\mathbf{1}$ and $\mathbf{4}$ on $\mathrm{Ag}(111)$; calculated adsorption geometries of $\mathbf{1}, \mathbf{3}$, and $\mathbf{4}$.

\section{ACKNOWLEDGMENTS}

T.J.T., S.U., T.M., R.H., and R.B. thank the Deutsche Forschungsgemeinschaft for financial support via SFB 677. I.P. and A.T. acknowledge financial support by the FNR-CORE project QUANTION.

\section{APPENDIX: EXPERIMENTAL AND THEORETICAL DETAILS}

\section{Synthesis of the molecules}

Functional groups were attached to the cationic TOTA platform 4 by polar $\mathrm{C}-\mathrm{C}$ and $\mathrm{C}-\mathrm{H}$ bond formation as described in the supplementary material in Sec. I. The powders 1-3 were purified by sublimation at $\approx 0.5 \mathrm{~Pa}$ and $\approx 100{ }^{\circ} \mathrm{C}$.

\section{Sample preparation}

Submonolayers of $\mathrm{TOTA}^{+} \mathrm{BF}_{4}^{-}$salt and the molecules 1-3 were sublimated onto flat and clean $\mathrm{Au}(111)$ held at ambient temperature. The sublimation parameters for molecules 1-3 were almost identical $\left(<10^{-7} \mathrm{~Pa}\right.$ and $\left.\approx 70^{\circ} \mathrm{C}\right)$ and milder than during the aforementioned purification by sublimation to make sure that intact molecules were deposited. The time between sublimation $(<2 \mathrm{~min}$ ) and cool down of the sample to $\approx 10 \mathrm{~K}$ for STM measurments did not exceed $25 \mathrm{~min}$.

\section{STM}

STM images were recorded at $4.6 \mathrm{~K},<10^{-9} \mathrm{~Pa}$, low constant currents (20-40 pA), and low positive sample voltages (100-130 mV). All STM topographs were acquired using the constant-current mode.

\section{DFT}

Electronic structure calculations were performed by using DFT with non-empirical exchange-correlation functional of Perdew-Burke-Ernzerhof ${ }^{62}$ (PBE) and the vdW ${ }^{\text {surf }}$ method to model screened van der Waals interactions for atoms and molecules on surfaces ${ }^{52-54}$ as implemented in the FHI-aims code $^{63}$ denoted PBE + vdW above.

The dissociation energies of reaction I (Table I) were obtained in two steps: $\mathrm{H}+\mathrm{R}-$ TOTA $\rightarrow \mathrm{H}-\mathrm{R}+$ TOTA $\rightarrow \mathrm{H}+\mathrm{R}+$ TOTA. The first step was calculated with DFT $+\mathrm{vdW}$. For the second step, experimental values of the dissociation energies of methane and ethyne measured at $298 \mathrm{~K}$, $4.55 \mathrm{eV}$, and $5.78 \mathrm{eV}$, were used. ${ }^{64}$ The dissociations II (Table I) were calculated in the same way but with $\mathrm{R}$ - TOTA and TOTA adsorbed on $\mathrm{Au}(111)$. All data for the reaction III (Table I) were obtained with DFT + vdW calculations. Finally, the dissociation energies of reaction IV (see Sec. III A) were determined in two steps: $2 \mathrm{R}-\mathrm{TOTA} / \mathrm{Au} \rightarrow 2$ $(\mathrm{R}+\mathrm{TOTA} / \mathrm{Au}) \rightarrow \mathrm{R}-\mathrm{R}+2$ TOTA/Au. The first step is reaction II for two isolated R - TOTA molecules on Au and was calculated as mentioned above. For the second step, experimental values of the bond formation energies of $\mathrm{H}_{3} \mathrm{C}-\mathrm{CH}_{3}$ and $\mathrm{H}_{2} \mathrm{C}-\mathrm{CH}_{2}$ measured at ambient temperature, $-3.91 \mathrm{eV}^{64}$ and $-6.51 \mathrm{eV},{ }^{50}$ were used.

The adsorption energies of $\mathbf{1}, \mathbf{3}$, and $\mathbf{4}$ (Table II) were directly calculated with the PBE + vdW and PBE functionals. The adsorption energies of the radicals were extracted from Table I as difference of the dissociation energies of the reactions II and III.

Further calculation details are given in the supplementary material in Sec. V.

\footnotetext{
${ }^{1}$ K. Ichimura, S. K. Oh, and M. Nakagawa, Science 288, 1624 (2000).

${ }^{2}$ E. Galoppini, W. Guo, W. Zhang, P. G. Hoertz, P. Qu, and G. J. Meyer, J. Am. Chem. Soc. 124, 7801 (2002).

${ }^{3}$ H. Jian and J. M. Tour, J. Org. Chem. 68, 5091 (2003).

${ }^{4}$ Q. Li, A. V. Rukavishnikov, P. A. Petukhov, T. O. Zaikova, C. Jin, and J. F. W. Keana, J. Org. Chem. 68, 4862 (2003).

${ }^{5}$ T. Kitagawa, Y. Idomoto, H. Matsubara, D. Hobara, T. Kakiuchi, T. Okazaki, and K. Komatsu, J. Org. Chem. 71, 1362 (2006).

${ }^{6}$ S. Wagner, F. Leyssner, C. Kördel, S. Zarwell, R. Schmidt, M. Weinelt, K. Rück-Braun, M. Wolfad, and P. Tegeder, Phys. Chem. Chem. Phys. 11, 6242 (2009)

${ }^{7}$ U. Siemeling, C. Bruhn, F. Bretthauer, M. Borg, F. Träger, F. Vogel, W. Azzam, M. Badin, T. Strunskus, and C. Wöll, Dalton Trans. 2009, 8593.

${ }^{8}$ K. Moth-Poulsen and T. Bjørnholm, Nat. Nanotechnol. 4, 551 (2009).

${ }^{9}$ F. von Wrochem, D. Gao, F. Scholz, H.-G. Nothofer, G. Nelles, and J. M. Wessels, Nat. Nanotechnol. 5, 618 (2010).
} 
${ }^{10}$ A. Schramm, C. Stroh, K. Dössel, M. Lukas, M. Fischer, F. Schramm, O. Fuhr, H. v. Löhneysen, and M. Mayor, Eur. J. Inorg. Chem. 2013, 70.

${ }^{11}$ W. Li, L. G. C. Rego, F.-Q. Bai, J. Wang, R. Jia, L.-M. Xie, and H.-X. Zhang, J. Phys. Chem. Lett. 5, 3992 (2014).

${ }^{12}$ E. Leary, A. L. Rosa, M. T. González, G. Rubio-Bollinger, N. Agraït, and N. Martín, Chem. Soc. Rev. 44, 920 (2015).

${ }^{13}$ R. Frisenda, S. Tarkuç, E. Galán, M. L. Perrin, R. Eelkema, F. C. Grozema, and H. S. J. van der Zant, Beilstein J. Nanotechnol. 6, 1558 (2015).

${ }^{14}$ Y. Ie, K. Tanaka, A. Tashiro, S. K. Lee, H. R. Testai, R. Yamada, H. Tada, and Y. Aso, J. Phys. Chem. Lett. 6, 3754 (2015).

${ }^{15}$ C. Schuschke, M. Schwarz, C. Hohner, T. N. Silva, L. Fromm, T. Döpper, A. Görling, and J. Libuda, J. Phys. Chem. Lett. 9, 1937 (2018).

${ }^{16}$ P. Du, M. Jaouen, A. Bocheux, C. Bourgogne, Z. Han, V. Bouchiat, D. Kreher, F. Mathevet, C. Fiorini-Debuisschert, F. Charra, and A.-J. Attias, Angew. Chem., Int. Ed. 53, 10060 (2014).

${ }^{17}$ M. Valášek and M. Mayor, Chem.-Eur. J. 23, 13538 (2017).

${ }^{18}$ B. Baisch, D. Raffa, U. Jung, O. M. Magnussen, C. Nicolas, J. Lacour, J. Kubitschke, and R. Herges, J. Am. Chem. Soc. 131, 442 (2009).

${ }^{19}$ Z. Wei, X. Wang, A. Borges, M. Santella, T. Li, J. K. Sørensen, M. Vanin, W. Hu, Y. Liu, J. Ulstrup, G. C. Solomon, Q. Chi, T. Bjørnholm, K. Nørgaard, and B. W. Laursen, Langmuir 30, 14868 (2014).

${ }^{20}$ T. Jasper-Tönnies, A. Garcia-Lekue, T. Frederiksen, S. Ulrich, R. Herges, and R. Berndt, Phys. Rev. Lett. 119, 066801 (2017).

${ }^{21}$ N. Hauptmann, L. Groß, K. Buchmann, K. Scheil, C. Schütt, F. L. Otte, R. Herges, C. Herrmann, and R. Berndt, New J. Phys. 17, 013012 (2015).

${ }^{22}$ F. Matino, G. Schull, F. Köhler, S. Gabutti, M. Mayor, and R. Berndt, Proc. Natl. Acad. Sci. U. S. A. 108, 961 (2010).

${ }^{23}$ N. L. Schneider, F. Matino, G. Schull, S. Gabutti, M. Mayor, and R. Berndt, Phys. Rev. B 84, 153403 (2011).

${ }^{24}$ S.-E. Zhu, Y.-M. Kuang, F. Geng, J.-Z. Zhu, C.-Z. Wang, Y.-J. Yu, Y. Luo, Y. Xiao, K.-Q. Liu, Q.-S. Meng, L. Zhang, S. Jiang, Y. Zhang, G.-W. Wang, Z.-C. Dong, and J. G. Hou, J. Am. Chem. Soc. 135, 15794 (2013).

${ }^{25}$ S. Yokoyama, T. Hirose, and K. Matsuda, Chem. Lett. 44, 1616 (2015).

${ }^{26}$ B. Hammer and J. K. Norskov, Nature 376, 238 (1995).

${ }^{27}$ A. Sanchez, S. Abbet, U. Heiz, W.-D. Schneider, H. Häkkinen, R. N. Barnett, and U. Landman, J. Phys. Chem. A 103, 9573 (1999).

${ }^{28}$ M. Haruta, Chem. Rec. 3, 75 (2003).

${ }^{29}$ M. Chen and D. Goodman, Science 306, 252 (2004).

${ }^{30}$ M. Chen, Y. Cai, Z. Yan and D. W. Goodman, J. Am. Chem. Soc. 128, 6341 (2006).

${ }^{31}$ A. S. K. Hashmi and G. J. Hutchings, Angew. Chem., Int. Ed. 45, 7896 (2006).

${ }^{32}$ B. Hvolbæk, T. V. Janssens, B. S. Clausen, H. Falsig, C. H. Christensen, and J. K. Nørskov, Nano Today 2, 14 (2007).

${ }^{33}$ H.-T. Chen, J.-G. Chang, S.-P. Ju, and H.-L. Chen, J. Phys. Chem. Lett. 1, 739 (2010).

${ }^{34}$ J. Gong, Chem. Rev. 112, 2987 (2011).

${ }^{35}$ For small molecules the binding energy was often used to define physisorption and chemisorption. This approach is not useful for largish molecule where dispersion interaction may be significant. For example, the calculated adsorption energy of 4-mercaptobenzoic acid on $\mathrm{Au}(111)$ of $1.75 \mathrm{eV}$ is mainly due to dispersion interaction $(1.2 \mathrm{eV}) .^{36}$

${ }^{36}$ N. Hauptmann, R. Robles, P. Abufager, N. Lorente, and R. Berndt, J. Phys. Chem. Lett. 7, 1984 (2016).
${ }^{37}$ P. Liljeroth, J. Repp, and G. Meyer, Science 317, 1203 (2007).

${ }^{38}$ S. Pan, Q. Fu, T. Huang, A. Zhao, B. Wang, Y. Luo, J. Yang, and J. Hou, Proc. Natl. Acad. Sci. U. S. A. 106, 15259 (2009).

${ }^{39}$ J. Repp, F. Moresco, G. Meyer, K.-H. Rieder, P. Hyldgaard, and M. Persson, Phys. Rev. Lett. 85, 2981 (2000).

${ }^{40}$ S. Lukas, G. Witte, and C. Wöll, Phys. Rev. Lett. 88, 028301 (2001).

${ }^{41}$ N. Knorr, H. Brune, M. Epple, A. Hirstein, M. A. Schneider, and K. Kern, Phys. Rev. B 65, 115420 (2002).

${ }^{42}$ K. Lau and W. Kohn, Surf. Sci. 75, 69 (1978).

${ }^{43} \mathrm{H}$. Brune, M. Giovannini, K. Bromann, and K. Kern, Nature 394, 451 (1998).

${ }^{44}$ K. Pohl, M. C. Bartelt, J. de la Figuera, N. C. Bartelt, J. Hrbek, and R. Q. Hwang, Nature 397, 238 (1999).

${ }^{45}$ S. Narasimhan and D. Vanderbilt, Phys. Rev. Lett. 69, 1564 (1992).

${ }^{46}$ I. Fernandez-Torrente, S. Monturet, K. J. Franke, J. Fraxedas, N. Lorente, and J. I. Pascual, Phys. Rev. Lett. 99, 176103 (2007).

${ }^{47}$ T. Knaak, T. G. Gopakumar, B. Schwager, F. Tuczek, R. Robles, N. Lorente, and R. Berndt, J. Am. Chem. Soc. 138, 7544 (2016).

${ }^{48}$ J. Kröger, H. Jensen, R. Berndt, R. Rurali, and N. Lorente, Chem. Phys. Lett. 438, 249 (2007).

${ }^{49}$ S. Soubatch, C. Weiss, R. Temirov, and F. S. Tautz, Phys. Rev. Lett. 102, 177405 (2009).

${ }^{50}$ S. W. Benson, J. Chem. Educ. 42, 502 (1965).

${ }^{51}$ M. Pan, D. W. Flaherty, and C. B. Mullins, J. Phys. Chem. Lett. 2, 1363 (2011).

${ }^{52}$ V. G. Ruiz, W. Liu, E. Zojer, M. Scheffler, and A. Tkatchenko, Phys. Rev. Lett. 108, 146103 (2012).

${ }^{53}$ R. J. Maurer, V. G. Ruiz, and A. Tkatchenko, J. Chem. Phys. 143, 102808 (2015).

${ }^{54}$ V. G. Ruiz, W. Liu, and A. Tkatchenko, Phys. Rev. B 93, 035118 (2016).

${ }^{55}$ G. Bistoni, P. Belanzoni, L. Belpassi, and F. Tarantelli, J. Phys. Chem. A 120, 5239 (2016).

${ }^{56}$ J. C. F. Rodriguez-Reyes, C. G. F. Siler, W. Liu, A. Tkatchenko, C. M. Friend, and R. J. Madix, J. Am. Chem. Soc. 136, 13333 (2014).

${ }^{57}$ S. Karakalos, Y. Xu, F. C. Kabeer, W. Chen, J. C. F. Rodríguez-Reyes, A. Tkatchenko, E. Kaxiras, R. J. Madix, and C. M. Friend, J. Am. Chem. Soc. 138, 15243 (2016).

${ }^{58}$ S. Ossinger, H. Naggert, L. Kipgen, T. Jasper-Toennies, A. Rai, J. Rudnik, F. Nickel, L. M. Arruda, M. Bernien, W. Kuch, R. Berndt, and F. Tuczek, J. Phys. Chem. C 121, 1210 (2017).

${ }^{59}$ Y. Xu, W. Chen, E. Kaxiras, C. M. Friend, and R. J. Madix, J. Phys. Chem. B 122, 555 (2017).

${ }^{60}$ F. C. Kabeer, W. Chen, R. J. Madix, C. M. Friend, and A. Tkatchenko, J. Phys. Chem. C 121, 27905 (2017).

${ }^{61}$ For example, the unit cell required for reliable adsorption geometries and energies contains 305 atoms (See supplementary material, Sec. V). This renders $a b$ initio calculations of reaction barriers with any reliable level of accuracy extremely challenging, if not impossible.

${ }^{62}$ J. P. Perdew, K. Burke, and M. Ernzerhof, Phys. Rev. Lett. 77, 3865 (1996).

${ }^{63}$ V. Blum, R. Gehrke, F. Hanke, P. Havu, V. Havu, X. Ren, K. Reuter, and M. Scheffler, Comput. Phys. Commun. 180, 2175 (2009).

${ }^{64}$ S. J. Blanksby and G. B. Ellison, Acc. Chem. Res. 36, 255 (2003). 